Pacific Journal of Mathematics

DESUSPENSIONS OF STUNTED PROJECTIVE SPACES 


\title{
DESUSPENSIONS OF STUNTED PROJECTIVE SPACES
}

\author{
DONALD M. DAVIS
}

\begin{abstract}
For multiples of the Hopf bundles over real and complex projective spaces, the number of sections is compared with the number of times that the Thom complex desuspends. Examples of equality of these numbers, and of inequality, are given.
\end{abstract}

1. Survey of Results. For $F$ the field of real numbers $R$ or complex numbers $C$, let $F P_{m}^{m+n}$ denote the stunted $F$-projective space $F P^{m+n} / F P^{m-1}$. In this paper, which combines some new results with a survey of known results, we consider the question of finding the largest $s$ such that $F P_{m}^{m+n}$ desuspends $s$ times, i.e. for which there exists a complex $Y$, often denoted $\Sigma^{-s} F P_{m}^{m+n}$, such that $\Sigma^{s} Y$ and $F P_{m}^{m+n}$ have the same homotopy type. This question is a good test for virtually any technique in (unstable) homotopy theory; e.g. secondary cohomology operations ([4]), Maunder operations ([5]), Adams operations in loop spaces ([12]), and unstable $B P$-operations ([36]). It is of particular importance because of its relation to the generalized vector field problem; since $F P_{m}^{m+n}$ is the Thom complex of $m \xi_{F, n}$, where $\xi_{F, n}$ denotes the Hopf bundle over $F P^{n}$, the following proposition is immediate (and very well known).

Proposition 1.1. If $m \xi_{F, n}$ has sections, then $\Sigma^{-s} F P_{m}^{m+n}$ exists.

These sectioning results are often conveniently expressed in terms of geometric dimension (gd), for which the desuspension analogue is the number $b\left(F P_{m}^{m+n}\right)$, where $b(X)$ is defined in $\S 2$. It is the minimal dimension of bottom cells of iterated desuspensions of iterated suspensons of $X$. We will show in 2.4 that for $X=F P_{m}^{m+m}$ the iterated suspensions can be omitted. Then 1.1 becomes

$$
\text { Proposition 1.1'. } b\left(F P_{m}^{m+n}\right) \leq \operatorname{gd}\left(m \xi_{F, n}\right) \text {. }
$$

The major theme of this paper will be to assess how strong 1.1 is, i.e. to determine which of the known nonsectioning results are implied by nondesuspensions. 
The simplest class of nonsectioning results consists of those implied by Stiefel-Whitney classes. These results all come from the nondesuspensions of the following result and $1.1^{\prime}$.

PROPOSITION 1.2. If $\left(\begin{array}{c}m \\ n\end{array}\right)$ is odd, then $b\left(R P_{m}^{m+n}\right) \geq n$ and $b\left(C P_{m}^{m+n}\right) \geq$ $2 n$.

Proof. In $H^{*}\left(R P_{m}^{m+n} ; Z_{2}\right), \mathrm{Sq}^{n}\left(x^{m}\right) \neq 0$. Since $\mathrm{Sq}^{n}$ vanishes on classes of degree less than $n, R P_{m}^{m+n}$ cannot desuspend below degree $n . C P_{m}^{m+n}$ is handled similarly using $\mathrm{Sq}^{2 n}$.

The other nonsectioning results obtained from ordinary cohomology are those of $m \xi_{C, n}$ implied by the Euler class.

Proposition 1.3. If $2 m \geq n$, then $\operatorname{gd}\left(m \xi_{C, n}\right) \geq n-\varepsilon$, where $\varepsilon=1$ if $n$ is odd and $\varepsilon=0$ if $n$ is even.

Proof. If $m \xi$ had the stable type of a bundle $\theta$ of dimension $n-\varepsilon$, then

$$
\chi(\theta)^{2}=p_{(n-\varepsilon) / 2}(\theta)=p_{(n-\varepsilon) / 2}(m \xi) \neq 0,
$$

and hence $\theta$ cannot have a section.

Many of these nonsectioning results are not implied by nondesuspensions, i.e. the Thom space desuspends lower than the bundle sections. Indeed, the nondesuspension analogue of 1.3 would say that no stunted complex projective space (SCP) can desuspend below the metastable range, where a complex with top dimension $T$ and bottom dimension $B$ is said to be metastable if $T \leq 3 B+2$. But this is patently false, because if $m \equiv 0\left(A_{n}\right)$, where $A_{n}$ is the Atiyah-Todd number, then $J\left(m \xi_{C, n}\right)=0$, and hence $b\left(C P_{m}^{m+n}\right)=0$. For further discussion, see $\S 2$.

A metastable example of $m \xi_{C, n}$ whose Thom complex desuspends lower than the bundle sections was communicated to the author by Mahowald in 1973. If $n$ is a 2-power, then $C P^{n} \subset R^{4 n-1}$ but $C P^{n} \nsubseteq R^{4 n-2}$, i.e. its immersion and embedding dimensions are equal ([22]). Thus the normal bundle to the embedding has no sections but its Thom complex desuspends ([8]). Although the normal bundle itself is probably not a multiple of the Hopf bundle, it is $J$-equivalent to $\left(A_{n}-n-1\right) \xi_{C, n}$. Since the fibers of $B O_{k} \rightarrow B O$ and $B G_{k} \rightarrow B G$ are equivalent in the stable range, it follows that $\operatorname{gd}\left(\left(A_{n}-n-1\right) \xi_{C, n}\right)=2 n-1$ and $b\left(C P_{A_{n}-n-1}^{A_{n}-1}\right) \leq$ $2 n-2$. 
The other main class of nonsectioning results for $C P$ 's is the following result, which has been proved by various applications of $K$-theory ([31], [13], [33], [24]).

Proposition 1.4. $\operatorname{gd}\left(m \xi_{C, n}\right) \geq 2 \cdot \max \left\{j-\nu_{2}\left(\left(\begin{array}{c}m \\ j\end{array}\right)\right): j \leq n\right\}$, where $\nu_{p}(N)$ is the exponent of $p$ in the factorization of $N$.

These results all come from nondesuspensions, using 1.1' and

THEOREM 1.5. $b\left(C P_{m}^{m+n}\right) \geq 2 \cdot \max \left\{j-\nu_{2}\left(\left(\begin{array}{c}m \\ j\end{array}\right)\right): j \leq n\right\}$.

This is proved by using the Adams operation $\psi^{2}$ in $K\left(C P_{m}^{m+n}\right)$. The result required is

THEOREM 1.6. $\psi^{k}$ is divisible by $k^{n+1}$ in $K\left(\Sigma^{2 n+1} Y\right)$.

If the " +1 "'s are omitted from 1.6, this follows from Adams' well-known formula $\psi^{k} \Sigma^{2}=k \Sigma^{2} \psi^{k}$. The full strength of 1.6 is proved in §3. In 1978 Sam Gitler and Steve Wilson independently pointed out to the author that the weaker form of 1.6 implied 1.5 within 1 dimension.

Using $\psi^{p}$ for odd primes $p$ in 1.6 , we obtain the following generalization of 1.5.

THEOREM 1.7.

$$
b\left(C P_{m}^{m+n}\right) \geq 2 \cdot \max \left\{j-\nu_{p}\left(\begin{array}{l}
m \\
j
\end{array}\right): p \text { prime, }(p-1) j \leq n\right\} .
$$

In $\S 3$ we present some discussion of when the odd primes in 1.7 improve upon the case $p=2$ (Theorem 1.5). A major difference is that the odd primes imply results only below the metastable range, and hence they imply no new nonsectioning results.

In view of the success which $K$-theory has traditionally enjoyed for complex projective spaces (e.g. [17], [27], [11]) it seems reasonable to conjecture that the lower bound for $b\left(C P_{m}^{m+n}\right)$ provided by 1.7 is sharp, i.e. all nondesuspensions of SCP's are detected by $\psi^{p}$ 's.

Conjecture 1.8.

$$
b\left(C P_{m}^{m+n}\right)=2 \cdot \max \left\{j-\nu_{p}\left(\begin{array}{l}
m \\
j
\end{array}\right): p \text { prime, }(p-1) j \leq n\right\} .
$$

Some evidence is provided by Theorem 3.6, which shows 1.8 is true when $n=2$, and by 3.8 , which shows that 1.8 is true when $b=0$. 
There are unstable Adams operations in $B P$-theory with properties analogous to those in $K$-theory. In Theorem 3.3 we show that the only nondesuspensions of SCP's which they imply are those (1.7) implied by $K$-theory. This furnishes additional evidence for Conjecture 1.8.

Very recently the author and Bendersky developed a method ([9]) of obtaining nondesuspension results using the coaction in BP-homology and unstable results of Ravenel-Wilson ([32]). These, too, yielded exactly 1.7 when applied to SCP's, again supporting Conjecture 1.8.

The situation when $F=R$ is, in at least one respect, quite different. Because $J\left(R P^{n}\right) \approx \tilde{K} O\left(R P^{n}\right)$, there are no quick-and-easy examples of $b\left(R P_{m}^{m+n}\right)<\operatorname{gd}\left(m \xi_{R, n}\right)$. Indeed, we give what appears to be the first example of this.

THEOREM 1.9. $\operatorname{gd}\left((64 k+36) \xi_{R, 10}\right)>5$, while $b\left(R P_{64 k+36}^{64 k+46}\right)=4$.

The second part of this result utilizes a delicate stable homotopy equivalence of [18]; Mahowald suggested to the author that the stable equivalences of [18] might provide a source for examples such as 1.9. The first part of 1.9 was proved by Adams in [2] using representation theory.

Two major families of nonsectioning results for $R P^{n}$ are known. The first ([10], [19], [15]) uses $K$-theory to prove: If $\left(\begin{array}{c}m-1 \\ n\end{array}\right)$ is odd, then $\operatorname{gd}\left(m \xi_{R, n}\right) \geq n-2 \nu_{2}(m)+\varepsilon$ where $\varepsilon$ depends upon $\bmod 4$ values of $n$ and $\nu_{2}(m)$ as in $[15 ; 2.1]$ or 1.10 below. In $\$ 5$ we prove the nondesuspension analogues.

THEOREM 1.10. If $\left(\begin{array}{c}m-1 \\ n\end{array}\right)$ is odd, then $b\left(R P_{m}^{m+n}\right) \geq n-2 \nu_{2}(m)+\varepsilon$, where $\varepsilon$ is given by the table:

\begin{tabular}{cr|rrrr}
\multicolumn{5}{c}{$n \bmod 4$} \\
$v(m) \bmod 4$ & 0 & 0 & 1 & 1 & 0 \\
\cline { 2 - 5 }$\nu$ & 1 & 1 & 1 & 2 & 1 \\
& 2 & 2 & 1 & 2 & 1 \\
3 & 2 & 1 & 0 & -1
\end{tabular}

This improves slightly upon the results of [12]. It answers Milgram's comment ([28; p. 10]): “it would be interesting to explain [10] in terms of obstructions to desuspension of Thom complex".

The other family of nonsectioning results for $R P$ is that proved by Astey ([7]) using BP. It has been recently proved in [9] that many of these are implied by nondesuspensions. 
2. Atiyah-Todd numbers. The $n$th Atiyah-Todd number $A_{n}$ is defined by $\nu_{p}\left(A_{n}\right)=\max \left\{j+\nu_{p}(j):(p-1) j \leq n\right\}$. It was proved in [3] that $J\left(m \xi_{C, n}\right)=0$ iff $m \equiv 0\left(A_{n}\right)$. Thus by [8] $C P_{m}^{m+n}=T\left(m \xi_{C, n}\right)$ and $C P_{0}^{n}$ are $S$-equivalent when $m \equiv 0\left(A_{n}\right)$, i.e. $\Sigma^{l} C P_{m}^{m+n}$ and $\Sigma^{l+2 m} C P_{0}^{n}$ have the same homotopy type for some $l$. As we show below, this implies that $C P_{m}^{m+n} \simeq \Sigma^{2 m} C P_{0}^{n}$, a fact used in $\S 1$.

Definition 2.1. If $X$ is a CW-complex, $b^{\prime}(X)$ is the dimension of the bottom cell of $\Sigma^{-s} X$ when $s$ is maximal.

REMARK 2.2. $b^{\prime}\left(\sum X\right) \leq b^{\prime}(X)$. An example in which these are unequal is $b^{\prime}\left(S^{n} \times S^{n}\right)=n, b^{\prime}\left(\Sigma\left(S^{n} \times S^{n}\right)\right)=0$.

DEFINITION 2.3. $b(X)=\lim _{s \rightarrow \infty} b^{\prime}\left(\Sigma^{s} X\right)$.

Proposition 2.4. $b^{\prime}\left(F P_{m}^{m+n}\right)=b^{\prime}\left(\sum^{l} F P_{m}^{m+n}\right)$ for all $m, n$, and $l$. Hence $b\left(F P_{m}^{m+n}\right)=b^{\prime}\left(F P_{m}^{m+n}\right)$.

Proof. Let $F=R$. A similar argument works if $F=C$. If $n \geq m$ then $b^{\prime}\left(R P_{m}^{m+n}\right)=b^{\prime}\left(\Sigma^{l} R P_{m}^{m+n}\right)=m$ since $\mathrm{Sq}^{m} x^{m} \neq 0$. If $n<m$ then $\Sigma^{l} R P_{m}^{m+n} d$ suspends uniquely to $R P_{m}^{m+n}$ for dimensional reasons (e.g. [28; 5.1]). Thus if $b=b^{\prime}\left(\Sigma^{l} R P_{m}^{m+n}\right)$, i.e.

$$
\Sigma^{l} R P_{m}^{m+n}=\Sigma^{m+l-b} X_{b}^{b+n}=\Sigma^{l}\left(\Sigma^{m-b} X_{b}^{b+n}\right),
$$

then $R P_{m}^{m+n}=\sum^{m-b} X_{b}^{b+n}$, and hence $b^{\prime}\left(R P_{m}^{m+n}\right)=b$.

3. Adams Operations. Theorem 1.6 follows from $\psi^{k} \Sigma^{2}=k \Sigma^{2} \psi^{k}$ together with

THEOREM 3.1. $\psi^{k}$ is divisible by $k$ in $\tilde{K}(\Sigma Y) \approx K^{1}(Y)$.

Proof (Hubbuck). Since products vanish in $\tilde{K}(\Sigma Y), 0=x^{p} \equiv \psi^{p} x$ $\bmod p$ for any prime $p([1])$. Write $k=p_{1}^{n_{1}} \cdots p_{r}^{n_{r}}$ with $p_{1}, \ldots, p_{r}$ distinct primes. Then $\psi^{k} x=\left(\psi^{p_{1}}\right)^{n_{1}} \cdots\left(\psi^{p_{r}}\right)^{r_{r}}(x)$ is divisible by $k$.

Proof of 1.7. $K\left(C P_{m}^{m+n}\right)$ is a free abelian group on generators $\alpha^{m}, \ldots, \alpha^{m+n}$. Let $M=\min \left\{i+\nu_{p}\left(\begin{array}{c}m \\ i\end{array}\right):(p-1)(m-i) \leq n\right\}=m-\max$, where max is as in 1.7. By 1.6 it suffices to show $\nu_{p}\left(\psi^{p} \alpha^{m}\right)=M$.

$$
\begin{aligned}
\psi^{p} \alpha^{m} & =\left((1+\alpha)^{p}-1\right)^{m}=\alpha^{m}\left(p \cdot g(\alpha)+\alpha^{p-1}\right)^{m} \\
& =\alpha^{m} \Sigma\left(\begin{array}{c}
m \\
i
\end{array}\right) p^{i} g(\alpha)^{l} \alpha^{(p-1)(m-l)}
\end{aligned}
$$


where $g(\alpha)=1+\cdots+\alpha^{p-2}$ with intermediate terms having integer coefficients. All terms have $\nu_{p}() \geq M$. If $i$ is the largest value for which this minimum is achieved, then $\nu_{p}\left(\right.$ coef of $\left.\alpha^{(p-1)(m-i)}\right)=M$, since only smaller $i$ can have any terms with $\nu_{p}()$ this small, but only larger $i$ can have any $\alpha^{(p-1)(m-i)}$-terms.

In order that the nondesuspension of 1.7 be in the metastable range, and hence imply a nonsectioning result not already implied by the Euler class, we would need $2\left(j-\nu_{p}\left(\begin{array}{c}m \\ j\end{array}\right)\right)>n$ for some $j$ with $(p-1) j \leq n$. This would imply $0>\nu_{p}\left(\begin{array}{c}m \\ j\end{array}\right)+(p-3) j$, which is clearly impossible if $p \geq 3$.

To illustrate the strength of the various $\psi^{p}$, we consider the lower bounds for $b\left(C P_{m}^{m+5}\right)$ implies by each. According to [17] there are 2880 distinct stable homotopy types of such spaces, and, as the table below illustates, $\psi^{3}$ is needed to give the best result for 70 of them, and $\psi^{5}$ for 4 of them; for the rest $\psi^{2}$ gives best results.

\begin{tabular}{c|c|l|r}
$\begin{array}{c}\text { Lower bound } \\
\text { for } b\left(C P_{m}^{m+5}\right)\end{array}$ & $\begin{array}{c}\text { Smallest } p \\
\text { Such that } \psi^{p} \\
\text { implies result }\end{array}$ & \multicolumn{1}{|c}{$\begin{array}{c}\text { Conditions } \\
\text { on } m\end{array}$} & \# of cases \\
\hline 10 & 2 & $5(8), 7(8)$ & 720 \\
8 & 2 & $4(8), 6(8), 9(16), 11(16)$ & 1080 \\
6 & 2 & $3(16), 8(16), 10(16), 17(32)$ & 630 \\
4 & 2 & $2(16), 16(32), 33(64)$ & 315 \\
4 & 3 & $32(96), 65(192)$ & 45 \\
2 & 2 & $1,96,129,160(192)$ & 60 \\
2 & 3 & $64(192), 192(576), 384(576)$ & 25 \\
2 & 5 & $576 k(2880), 1 \leq k \leq 4$ & 4 \\
0 & 2 & $0(2880)$ & 1
\end{tabular}

Novikov ([30]) introduced Adams operations $\psi^{k}$ in rational $M U$-theory and Araki ([6]) showed that they are also defined in rational $B P$-theory. Wilson ([36]) showed that $k^{i} \psi^{k}$ is integral on $M U^{2 i+\varepsilon}(X)$ and $B P^{2 i+\varepsilon}(X)$ for $\varepsilon=0$ or 1 , but was not optimistic about their utility. We reinforce his opinion, and our Conjecture 1.8, in the following theorem.

THEOREM 3.3. The only nondesuspensions of SCP's implied by the $B P$-Adams operations are those already implied by the KU-Adams operations.

Proof. It suffices to consider $\psi^{p}$ in $B P Q$, localized at $p . B P^{*}=$ $Z_{(p)}\left[v_{l}: i \geq 1\right]$, and $B P^{*}\left(C P_{m}^{m+n}\right)$ is a free $B P^{*}$-module with generators 
$\alpha^{m}, \ldots, \alpha^{m+n}$. In $B P^{*}\left(C P^{\infty}\right)$

$$
\begin{aligned}
\psi^{p}(\alpha) & =\frac{1}{p}[p](\alpha)=\frac{1}{p}\left(p \alpha-\left(p^{p-1}-1\right) v_{1} \alpha^{p}+\cdots\right) \\
& =\frac{1}{p} \alpha \sum_{i=0}^{\infty} c_{i} \alpha^{(p-1) i}
\end{aligned}
$$

where $c_{0}=p, c_{1}=\left(1-p^{p-1}\right) v_{1}$, and $c_{i}$ is an integer combination of $v_{i}$ 's, since the $p$-series $[p](\alpha)$ is induced by a map $C P^{\infty} \rightarrow C P^{\infty}$.

Then

$$
\psi^{p}\left(\alpha^{m}\right)=\alpha^{m} \cdot \sum_{j \leq[n /(p-1)]} \alpha^{(p-1) j} C_{j},
$$

where

$$
C_{j}=\sum \frac{1}{p^{i_{1}+\cdots+i_{r}}} \frac{m !}{i_{1} ! \cdots i_{r} !\left(m-i_{1}-\cdots i_{r}\right) !} c_{1}^{i_{1}} \cdots c_{r}^{i_{r}}
$$

this sum taken over tuples $\left(i_{1}, \ldots, i_{r}\right)$ of nonnegative integers with $i_{1}+2 i_{2}$ $+\cdots+r i_{r}=j$ and $i_{1}+\cdots+i_{r} \leq m . C_{j}$ can be written as a polynomial in the $v_{i}$ 's, and $\nu_{p}\left(C_{j}\right)$ means $\nu_{p}$ (this polynomial in the $v_{i}$ 's). We will show below that

(3.4) $\min \left\{\nu_{p}\left(C_{j}\right): j \leq\left[\frac{n}{p-1}\right]\right\}=\min \left\{\nu_{p}\left(\begin{array}{c}m \\ j\end{array}\right)-j: j \leq\left[\frac{n}{p-1}\right]\right\}$,

and this together with Wilson's integrality result mentioned above implies exactly the nondesuspensions of 1.7 by a similar argument.

To see (3.4), we note that

$$
\nu_{p}\left(\operatorname{coef} \text { of } c_{1}^{i_{1}} \cdots c_{r}^{i_{r}}\right) \geq \nu_{p}\left(\operatorname{coef} \text { of } c_{1}^{i_{1}+\cdots+i_{r}}\right)=\nu_{p}\left(\begin{array}{c}
m \\
j
\end{array}\right)-j,
$$

which immediately implies $\geq$ in (3.4). Choose the smallest $j$ realizing the minimum value, $M$, of $\nu_{p}\left(\begin{array}{c}m \\ j\end{array}\right)-j$. The coefficient of $v_{1}^{j}$ contains a term

$$
\left(\begin{array}{c}
m \\
j
\end{array}\right)\left(\frac{p^{p-1}-1}{p}\right)^{j}
$$

from $c_{1}^{j}$ with $\nu_{p}()=M$. Other terms $c_{1}^{k_{1}} \cdots c_{r}^{k_{r}}$ contributing $v_{1}^{j}$-terms have $\nu_{p}\left(\right.$ coef of $\left.v_{1}^{j}\right)>M$, by (3.5), since $k_{1}+\cdots+r k_{r}=j$ and so $k_{1}+\cdots+k_{r}$ $<j$. Therefore $\nu_{p}\left(\right.$ coef of $\left.v_{1}^{j}\right)=M$.

The next result verifies Conjecture 1.8 when $n=2$ by showing that the Adams operations detect all nondesuspensions of SCP's with three cells. 
THEOREM 3.6.

$$
b\left(C P_{m}^{m+2}\right)=2 \cdot \max \left\{1-\nu_{2}(m), 2-\nu_{2}\left(\begin{array}{c}
m \\
2
\end{array}\right), 1-\nu_{3}(m), 0\right\} .
$$

Proof. By [17] there are 14 stable homotopy types of SCP's, which depend upon the mod 24 value of $m$. The required values of $b$ are listed below (where " $="$ is from [17], e.g. $C P_{24 k+1}^{24 k+3} \simeq{ }_{s} C P_{24 l+17}^{24 l+19}$ ):

\begin{tabular}{l|l}
$b$ & $m \bmod 24$ \\
\hline 0 & 0 \\
2 & $1=17, \quad 4=20, \quad 5=13, \quad 8=16,9,12,21$ \\
4 & $2=22, \quad 3=15, \quad 6=18, \quad 7=11, \quad 10=14, \quad 19=23$
\end{tabular}

The $\geq$-part of this result is 1.7 ; the work required here is to show that these SCP's desuspend as far as claimed. The case $m \equiv 0$ was handled in $\S 2$. The cases in which $b=4$ are implied for dimensional reasons, since $\Sigma^{2 m-4} \Omega^{2 m-4} C P_{m}^{m+2} \rightarrow C P_{m}^{m+2}$ is a $(2 m+4)$-equivalence.

The four cases $m$ odd, $b=2$ are handled by noting that

$$
\Sigma^{2 m-2} C P^{2} \simeq C P_{m}^{m+1} \text { and } \pi_{5}\left(C P^{2}\right) \stackrel{\Sigma^{2 m-2}}{\rightarrow} \pi_{2 m+3}\left(C P_{m}^{m+1}\right)
$$

is a surjection $Z \rightarrow Z_{12}$; thus the attaching map for the top cell of $C P_{m}^{m+2}$ is the image of $\alpha \in \pi_{5} C P^{2}$, and so $C P_{m}^{m+2} \simeq \Sigma^{2 m-2}\left(C P^{2} \cup_{\alpha} e^{6}\right)$.

The cases $m \equiv 4$ and 12 can be proved using James' cell decomposition of $\Omega S^{3}=S^{2} \cup e^{4} \cup e^{6} \cup \cdots . \pi_{5}\left(\Omega S^{3}\right) \approx Z_{12}$ and the generator $\alpha$ stabilizes to $2 g+\eta \in \pi_{n+3} S^{n} \oplus \pi_{n+3} S^{n+2}$. ( $\eta$ is present by consideration of the Hopf invariant $H: \pi_{5} \Omega S^{3} \rightarrow \pi_{5} \Omega S^{5}$.)

$$
\Sigma^{2 m-2}\left(\Omega S^{3}\right)^{(4)} \simeq S^{2 m} \vee S^{2 m+2} \simeq C P_{m}^{m+1},
$$

and

$$
\pi_{5}\left(\Omega S^{3}\right)^{(4)} \stackrel{\Sigma^{2 m-2}}{\rightarrow} \pi_{2 m+3} C P_{m}^{m+1}=Z_{24} \oplus Z_{2}
$$

has image all multiples of $2 g+\eta$. The attaching map for the top cell of $C P_{m}^{m+2}$ is of this form: $\eta$ is present since $\mathrm{Sq}^{2}$ is nonzero, and the attaching map to the bottom cell is repsectively $2 g$ or $6 g$ as can be seen by considering $\mathrm{CP}_{m}^{m+2} \rightarrow Q P_{m / 2}^{m / 2+1}=T(m H / 2)$.

Finally we prove it for $m \equiv 8$ by showing

$$
\pi_{5}\left(S^{2} \cup_{4 h} e^{4}\right) \stackrel{\Sigma^{2 m-2}}{\rightarrow} \pi_{2 m+3}\left(S^{2 m} \vee S^{2 m+2}\right) \approx Z_{24} \oplus Z_{2}
$$


has in its image the class $4 g_{1}+\eta$, which is the attaching map for the top cell of $C P_{m}^{m+2}$. It suffices to consider the single suspension and show

$$
\pi_{5}\left(S^{2} \cup_{4 h} e^{4}\right) \stackrel{\Sigma}{\rightarrow} \pi_{6}\left(S^{3} \vee S^{5}\right) \approx Z_{12}+Z_{2}
$$

has in its image the class $2 g+\eta$. If we let $J()=\Omega \Sigma($ ) and use the homomorphism of [35, p. 547], the result follows from

LEMma 3.7. If $X=S^{2} \cup_{4 h} e^{4}$, then in

$$
\begin{array}{lc}
\pi_{6}\left(S^{3} \vee S^{5}\right)=\pi_{5}(J X) \stackrel{i_{*}}{\rightarrow} \pi_{5}(J X, X) \stackrel{\bar{h}_{*}}{\rightarrow} \pi_{5}(J(X \wedge X)) \\
Z_{12} \oplus Z_{2} & Z_{2}
\end{array}
$$

(i) $\bar{h}_{*} i_{*}(\eta)=0\left(\right.$ and hence $\left.\bar{h}_{*} i_{*}(2 g+\eta)=0\right)$ and

(ii) $\operatorname{ker}\left(\bar{h}_{*}\right)$ is torsion free.

Proof. (i) The 5-skeleton $(J X)^{(5)}$ is $S^{2} \cup_{4 h} e^{4} \cup_{2 h} e^{4}$. There is an equivalence $\left(S^{2} \cup_{2 g} e^{4}\right) \vee S^{4} \rightarrow(J X)^{(5)}$ which sends the split $S^{4}$ to the first 4-cell by degree 1 and to the second 4-cell by degree -2 . The class $\eta$ under consideration is the image of the nonzero element of $\pi_{5} S^{4}$ in $\pi_{5}(J X)$ (since $Z_{12} \oplus Z=\pi_{5}\left(S^{2} \cup_{2 g} e^{4}\right) \rightarrow \pi_{5} J X=Z_{12}$ sends the $Z$ to 0 ). When $X$ is collapsed, we have $\pi_{5} S^{4} \stackrel{-2}{\rightarrow} \pi_{5} S^{4}$, which is 0 .

(ii) $\bar{h}_{*}$ can be factored into $\pi_{5}(J X, X) \rightarrow \pi_{5}(J X / X) \rightarrow \pi_{5}(J(X \wedge X))$. The second homomorphism is injective since through dimension 6 it is a compression $J(X \wedge X) \cup e^{6} \rightarrow J(X \wedge X)$. The first has torsion-free kernel by standard stability arguments since $X$ and $J X$ have only even-dimensional cells and $J X / X$ is 3-connected. [Let $F=$ fibre $(X \rightarrow J X)$. The Serre spectral sequence shows $H^{5}(F)$ torsion-free. (Everything below it is torsion-free, so a torsion class could not support a nonzero differential, but $H^{5} X=0$.) Therefore $H_{5}(\Sigma F)$ is torsion-free, hence so is $H_{6}$ (cofibre $(\Sigma F \rightarrow J X / X))$, and thus also $\pi_{5}($ fibre $(\Sigma F \rightarrow J X / X))$.

The following result provides some evidence for Conjecture 1.8 by showing that in all cases in which the Adams operations merely show $b \geq 0$, we do indeed have $b=0$.

Proposition 3.8. $J\left(m \xi_{C, n}\right)=0$ iff

$$
\max \left\{j-\nu_{p}\left(\begin{array}{c}
m \\
j
\end{array}\right): \text { p prime, }(p-1) j \leq n\right\}=0 \text {. }
$$


Proof. By [3] we are reduced to showing, if $p, m$, and $J$ are fixed, then $\nu_{p}(m) \geq j+\nu_{p}(j)$ for all $j \leq J$ iff $\nu_{p}\left(\begin{array}{c}m \\ j\end{array}\right) \geq j$ for all $j \leq J$. This follows easily from (i)

$$
\left(\begin{array}{c}
m \\
j
\end{array}\right)=\frac{m}{j}\left(\begin{array}{c}
m-1 \\
j-1
\end{array}\right)
$$

and (ii) $j \leq p^{r} \mid m$ implies $\left(\begin{array}{c}m-1 \\ j-1\end{array}\right) \not 0(p)$.

4. Example of $b\left(R P_{m}^{m+n}\right)<\operatorname{gd}\left(m \xi_{R, n}\right)$. In this section we prove Theorem 1.9.

Proof that $b\left(R P_{64 k+36}^{64 k+46}\right)=4$. $\mathrm{Sq}^{4}$ will not allow it to desuspend below 4. The desuspension to 4 follows from 2.1 and the fact ([18]) that $P_{64 k+36}^{64 k+46}$ and $P_{4}^{14}$ have the same stable homotopy type. The result there is stated only for SRP's with at least 13 cells; moreover, this case would use their Lemma 3.6, of which only the barest hint of proof is given. So we prove it.

Since $J\left(R P^{10}\right) \approx Z_{64}$, it suffices to prove it in the single case $P_{100}^{110}$. Since $32 \xi_{9}$ is trivial, the classifying map for $100 \xi_{10}$ can be factored as:

$$
\begin{aligned}
& P_{10} \stackrel{\Delta}{\rightarrow} P^{10} \times \underbrace{10} \stackrel{1 \times p}{\rightarrow} P^{10} \times S^{10} \rightarrow B O_{68} \times B O_{32} \rightarrow B O_{100}, \\
& 68 \xi \times 32 \xi
\end{aligned}
$$

and the induced map of Thom spaces is

$$
P_{100}^{110} \rightarrow P_{68}^{78} \wedge\left(S^{32} \cup_{\eta^{2} \sigma} e^{42}\right)
$$

which compresses into the 110-skeleton $\Sigma^{32} P_{68}^{78} \cup_{\eta^{2} \sigma} e^{110}$. By [25, p. 57] the image of $\eta^{2} \sigma$ in $\pi_{77}\left(S^{68}\right) \stackrel{i_{*}}{\rightarrow} \pi_{77}\left(P_{68}^{78}\right)$ has filtration $\geq 4$, and multiplication by $\eta^{2}$ is injective on classes of $\pi_{77}\left(P_{68}^{78}\right)$ of filtration $\geq 4$. Since $\eta^{4}=0$, this implies $i_{*}\left(\eta^{2} \sigma\right)=0$. Therefore $P_{68}^{78} \cup_{\eta^{2} \sigma} e^{78} \simeq P_{68}^{78} \vee S^{78}$, and the following composite is an equivalence:

$$
\begin{aligned}
P_{100}^{110} & \rightarrow \Sigma^{32} P_{68}^{78} \cup_{\eta^{2} \sigma} e^{110} \simeq \Sigma^{32} P_{68}^{78} \vee S^{110} \stackrel{p}{\rightarrow} \Sigma^{32} P_{68}^{78} \\
& \simeq \Sigma^{96} P_{4}^{14} .
\end{aligned}
$$

5. Nondesuspensions of SRP's implied by $J$. In this section we prove Theorem 1.10. We use the method of [28; 5.1]. In this section $\nu(m)=\nu_{2}(m)$. Let $d=m-n+2 \nu(m)-\varepsilon+1$. If $P_{m}^{m+n}$ desuspends $d$ 
times, the dotted line in the diagram

$$
\begin{aligned}
& \Sigma^{-d} \Sigma^{-1} P_{m+1}^{m+n}---\rightarrow S^{m-d} \\
& \Omega^{d} \Sigma^{-1} P_{m+1}^{m+n} \stackrel{\Omega^{d} \alpha}{\longrightarrow} \Omega^{d} S^{m} \\
& \downarrow s \\
& Q\left(\Sigma^{m-d} P_{m-d}\right)
\end{aligned}
$$

can be filled in, where $\alpha$ is the attaching map, $Q=\Omega^{\infty} \Sigma^{\infty}$, and $s$ is the Snaith map ([34]). Thus it suffices to prove nontriviality of the composite

$$
P_{m+1}^{m+n} \rightarrow \Sigma^{d+1} \Omega^{d} S^{m} \rightarrow \Sigma^{d+1} Q\left(\Sigma^{m-d} P_{m-d}\right) \rightarrow \Sigma^{m+1} P_{m-d} \wedge J,
$$

where $J$ is the spectrum called $b J$ in $[15 ; \S 2]$ and $J$ in [26].

The attaching map $\alpha$ factors through $P_{m+\rho}^{m+n}$, where $\rho=\rho(m)=8 a+$ $2^{b}$ if $\nu=\nu(m)=4 a+b$ with $0 \leq b \leq 3$. The class of (5.1) lies in the middle group of the exact sequence

$$
\begin{gathered}
\stackrel{\psi^{3}-1}{\rightarrow}\left[P_{m+\rho}^{m+n}, \Sigma^{m+1} P_{m-d} \wedge \Sigma^{3} b s p\right] \stackrel{\delta}{\rightarrow}\left[P_{m+\rho}^{m+n}, \Sigma^{m+1} P_{m-d} \wedge J\right] \\
\rightarrow\left[P_{m+\rho}^{m+n}, \Sigma^{m+1} P_{m-d} \wedge b o\right]^{\psi^{3}-1} \rightarrow
\end{gathered}
$$

which is calculated as in $[15 ;$ p. 42]. We will prove

Proposition 5.2. The class of (5.1) is the image under $\delta$ of a class of filtration $\nu(m)-3$.

This is nontrivial exactly as in [15; §2], establishing 1.10.

To do this, we consider the following prototype diagram, whose right vertical composite is $(5.1)$.

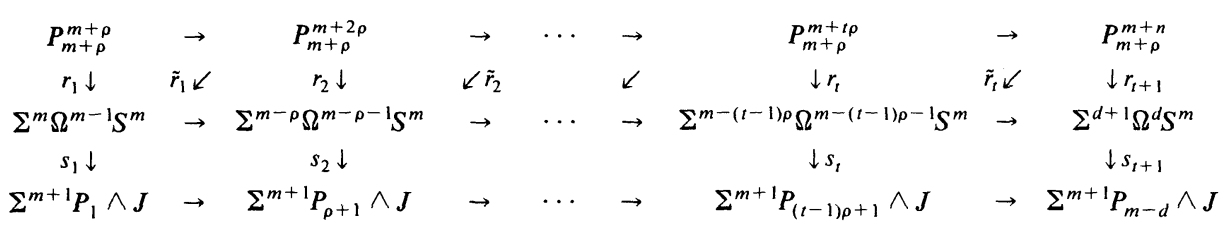

Here $t=[n / \rho]$ and $\tilde{r}_{j}$ is the composite

$$
\Sigma^{k} \Sigma^{-k} P_{m+\rho}^{m+(j+1) \rho} \rightarrow \Sigma^{k} \Omega^{k+1} \Sigma^{-1} P_{m+\rho}^{m+(j+1) \rho} \stackrel{\Sigma^{k} \Omega^{k+1} \alpha}{\rightarrow} \Sigma^{k} \Omega^{k+1} S^{m}
$$


where $k=m-(j-1) \rho-1$, the desuspension existing for dimensional reasons.

We make the following minor modifications in (5.3):

If $\rho \equiv 1(8)$, replace $j \rho$ by $j(\rho-1)$ in bottom two rows and by $j(\rho-1)+2$ in top row when $j \geq 2$.

If $\rho \equiv 2(8)$, add 1 to top dimensional $m+j \rho$ in the top row when $j$ is even.

If $\rho \equiv 0(4)$, replace $j \rho$ by $j(\rho-2)+2$ throughout the diagram, and replace bottom dimension of spaces in top row by $m+\rho-1$.

By $[29 ; 5.4 .1], r_{1}$ is a generator of the image of $J$, and so [26; 8.3] implies that the left vertical composite is the image under $\delta$ of a class of filtration $\nu(m)-3$, where $\delta$ is as in 5.2. The induction step uses the prototype diagram

$$
\left[P_{m+\rho}^{m+(j+1) \rho}, \Sigma^{m+1} P_{(j-1) \rho+1} \wedge J\right] \stackrel{c_{*}}{\rightarrow}\left[P_{m+\rho}^{m+(j+1) \rho}, \Sigma^{m+1} P_{j \rho+1} \wedge J\right]
$$

$$
\left[P_{m+\rho}^{m+j \rho}, \Sigma^{m+1} P_{(j-1) \rho+1} \wedge J\right]
$$

modified as above. The homomorphisms are surjections of groups in $\operatorname{im}(\delta)$ :

$$
\begin{aligned}
& \mathbf{Z} / 2^{2 \nu-2} \stackrel{c_{*}}{\rightarrow} \mathbf{Z} / 2^{\nu-2} \\
& \quad \downarrow_{l *} \\
& \mathbf{Z} / 2^{\nu-2}
\end{aligned}
$$

We ignore here low filtration $\mathbf{Z}_{2}$ 's, which can be shown by naturality to play no significant role. The order of the $\mathbf{Z} / 2^{2 \nu-2}$ is only approximate; the important part is that $2^{\nu-3}$ is nonzero in all groups.

(5.3) implies that in (5.4)

$$
\begin{aligned}
& {\left[s_{j} \tilde{r}_{j}\right] \mapsto\left[s_{J+1} r_{J+1}\right]} \\
& \quad \downarrow \\
& {\left[s_{j} r_{j}\right]}
\end{aligned}
$$

The induction hypothesis is that $\left[s_{j} r_{j}\right]$ is nonzero (in fact, is $2^{\nu-3}$ times a generator), and (5.5) and (5.6) then imply the same of $\left[s_{J+1} r_{j+1}\right]$, proving 5.2 and hence 1.10 . 
In [15] (see also [16]), sectioning results were obtained which showed that for certain $n$ and $m\left(n / 4+4 \log _{2}(n / 2-\nu(m)+4)+11 \leq \nu(m) \leq\right.$ $n / 2-75)$, the nonsectioning results implied by 1.10 are best possible. In these cases we have $\operatorname{gd}\left(m \xi_{n}\right)=b\left(P_{m}^{m+n}\right)=n-2 \nu(m)+\varepsilon$; in particular, the gd and $b$ here depend only upon $n$ and $\nu(m)$. This is not always true when $n$ and $m$ do not satisfy these conditions.

One case of particular interest concerns $2^{i} \xi_{2^{i}-1}$ and $\left(2^{L}-2^{i}\right) \xi_{2^{i}-1}$, which are stably the tangent bundle and normal bundle of $R P^{2^{i}-1}$. Since the number of vector fields on $R P^{n}$ equals the number on $S^{n}$, we have $\operatorname{gd}\left(2^{i} \xi_{2^{i}-1}\right)=2^{i}-2 i-\Delta$ where

$$
\Delta=\left\{\begin{array} { l } 
{ 1 } \\
{ 0 } \\
{ 2 }
\end{array} \text { if } i \equiv \left\{\begin{array}{l}
0 \\
1,2(4) \\
3
\end{array} \quad([1]) .\right.\right.
$$

James ([23]) showed $\operatorname{gd}\left(\left(2^{L}-2^{i}\right) \xi_{2^{i}-1}\right) \geq 2^{i}-2 i-\varepsilon$, and Gitler and Mahowald ([20], [21]) presented an argument which purported to show $\operatorname{gd}\left(\left(2^{L}-2^{i}\right) \xi_{2^{i}-1}\right)=\operatorname{gd}\left(2^{i} \xi_{2^{i}-1}\right)$ by showing that the relevant map of classifying spaces is an $H$-map. In $1979 \mathrm{Crabb}$ and Steer noted a mistake in the argument of [21], so that $\operatorname{gd}\left(\left(2^{L}-2^{i}\right) \xi_{2^{i}-1}\right)$ is still an open question. Of course this is equivalent to determining the immersion dimension of $R P^{2^{i}-1}$. The situation of $2^{i} \xi_{2^{i}-1}$ is rather unusual in that the stable geometric dimension of [15] is already realized, i.e. $\operatorname{gd}\left(2^{i} \xi_{2^{i}-1}\right)=$ $\operatorname{gd}\left(2^{i+4 k} \xi_{2^{i}+8 k-1}\right)$ for all $k$. For large $k$ the bundle is defined on a stable complex, and so multiplication of the bundle by an odd number doesn't change its gd. If this were true for odd multiples of $2^{l} \xi_{2^{i}-1}$, then the desired immersion of $R P^{2^{i}-1}$ would follow immediately, but here it is not clear.

\section{REFERENCES}

[1] J. F. Adams, Vector fields on spheres, Ann. of Math., 75 (1962), 603-632.

[2] _ Geometric dimension of bundles over $R P^{n}$, Proc. of Int. Conf. on Prospects in Math., Research Inst. for Math. Sci., Kyoto Univ. 1973, 1-17.

[3] J. F. Adams and G. Walker, On complex Stiefel manifolds, Proc. Camb. Phil Soc., 61 (1965), 81-103.

[4] J. Adem and S. Gitler, Non-immersion theorems for real projective spaces, Bol. Soc. Mat. Mex., 9 (1964), 37-50.

[5] J. Adem and K. Y. Lam, Evaluation of Some Maunder Cohomology Operations, Lecture Notes in Math., Springer Verlag, 597 (1977), 1-31.

[6] S. Araki, Multiplicative operations in BP cohomology, Osaka J. Math., 12 (1975), 343-356. 
[7] L. Astey, Geometric dimension of bundles over real projective spaces, Quar. J. Math Oxford, 31 (1980), 139-155.

[8] M. F. Atiyah, Thom complexes, Proc. London Math. Soc., 11 (1961), 291-310.

[9] M. Bendersky and D. Davis, Unstable BP-homology and desuspensions, to appear in Amer. J. Math.

[10] D. M. Davis, Generalized homology and the generalized vector field problem, Quar. J. Math. Oxford, 25 (1974), 169-193.

[11] BP-operations and Maps of Stunted Complex Projective Spaces, Proc. Waterloo Topology Conf., Lecture Notes in Math., Springer-Verlag, 741 (1979), 373-393.

[12] D. M. Davis and M. Mahowald, A nondesuspension theorem for stunted real projective spaces, Proc. Amer. Math. Soc., 7 (1978), 143-146.

[13] Immersions of complex projective spaces and the generalized vector field problem, Proc. London Math. Soc., 35 (1977), 333-348.

[14] Obstruction Theory and ko-theory, Proc. Evanston Homotopy Theory Conference, Lecture Notes in Math., Springer-Verlag, 658 (1978), 134-164.

[15] D. M. Davis, S. Gitler and M. Mahowald, The stable geometric dimension of vector bundles over real projective spaces, Trans. Amer. Math. Soc., 268 (1981), 39-62.

[16] _ Corrections to 'Stable geometric dimension of vector bundles over real projective spaces, Trans. Amer. Math. Soc., 280 (1983), 841-843.

[17] S. Feder and S. Gitler, The classification of stunted projective spaces by stable homotopy type, Trans. Amer. Math. Soc., 225 (1977), 59-81.

[18] S. Feder, S. Gitler and M. Mahowald, On the stable homotopy type of stunted projective spaces, Bol. Soc. Mat. Mex., 22 (1977), 1-5.

[19] S. Feder and W. Iberkleid, Secondary Operations in K-theory and the Generalized Vector Field Problem, Lecture Notes in Math, Springer-Verlag, 597 (1977), 161-175.

[20] S. Gitler and M. Mahowald, The immersion of manifolds, Bull. Amer. Math. Soc., 73 (1967), 696-700.

[21] Some immersions of real projective spaces, Bol. Soc. Mat. Mex., 14 (1969), 9-21.

[22] I. M. James, Euclidean models of projective spaces, Bull. London Math. Soc., 3 (1971), 257-276.

[23] _ On the immersion problem for real projective spaces, Bull. Amer. Math. Soc., 69 (1963), 231-238.

[24] B. Lawson and M. L. Michelson, Clifford bundles, immersions of manifolds, and the vector field problem, J. Differential Geom., 15 (1981), 237-267.

[25] M. Mahowald, The metastable homotopy of $S^{n}$, Mem. Amer. Math. Soc., 72 (1967).

[26] _ The image of $J$ in the EHP sequence, Ann. Math., 116 (1982), 65-112.

[27] C. McGibbon, Self-maps of projective spaces, Trans. Amer. Math. Soc., 271 (1982), 325-346.

[28] R. J. Milgram, Unstable Homotopy From the Stable Point of View, Lecture Notes in Math., 368 (1974) Springer-Verlag.

[29] _ Group representations and the Adams spectral sequence, Pacific J. Math., 41 (1972), 157-182.

[30] S. P. Novikov, The methods of algebraic topology from the point of view of cobordism theories, Math. U.S.S.R. Izvestya, 1 (1967), 827-913.

[31] D. Randall, Note on the generalized vector field problem, Bol. Soc. Mat. Mex., 17 (1972), 40-41.

[32] D. C. Ravenel and W. S. Wilson, The Hopf-ring for complex cobordism, J. Pure Appl. Algebra, 9 (1977), 241-280.

[33] V. P. Snaith, The geometric dimension of complex vector bundles, Northwestern Homotopy Theory Conf., Soc. Mat. Mex., (1974), 199-226. 
[34] A stable decomposition of $\Omega^{n} S^{n} X$, J. London Math. Soc., 7 (1974), 577-583.

[35] G. W. Whitehead, Elements of Homotopy Theory, Graduate texts of mathematics, Springer-Verlag, 1978.

[36] W. S. Wilson, A BP introduction and sampler, CBMS Regional Conf. Series 48 (1982).

Received March 3, 1982. The author was partially supported by an N.S.F. Research Grant and partially supported by an S.R.C. research grant while at the University of Warwick.

\section{LEHIGH UNIVERSITY}

BETHLEHEM, PA 18015 



\section{PACIFIC JOURNAL OF MATHEMATICS \\ EDITORS}

Donald BABBITT (Managing Editor)

University of California

Los Angeles, CA 90024

Hugo Rossi

University of Utah

Salt Lake City, UT 84112

C. C. Moore and Arthur Ogus

University of California

Berkeley, CA 94720
J. DugunduI

Department of Mathematics

University of Southern California

Los Angeles, CA 90089-1113

R. FinN and H. SAMELSON

Stanford University

Stanford, CA 94305

ASSOCIATE EDITORS

R. ARENS

E. F. BECKENBACH

B. H. NeUMANN

F. WOLF

K. YosHIDA (1906-1982)

\section{SUPPORTING INSTITUTIONS}

UNIVERSITY OF ARIZONA

UNIVERSITY OF BRITISH COLUMBIA

CALIFORNIA INSTITUTE OF TECHNOLOGY

UNIVERSITY OF CALIFORNIA

MONTANA STATE UNIVERSITY

UNIVERSITY OF NEVADA, RENO

NEW MEXICO STATE UNIVERSITY

OREGON STATE UNIVERSITY
UNIVERSITY OF OREGON

UNIVERSITY OF SOUTHERN CALIFORNIA

STANFORD UNIVERSITY

UNIVERSITY OF HAWAII

UNIVERSITY OF TOKYO

UNIVERSITY OF UTAH

WASHINGTON STATE UNIVERSITY

UNIVERSITY OF WASHINGTON 


\section{Pacific Journal of Mathematics}

\section{Vol. 113, No. $1 \quad$ March, 1984}

Flavio E. A. da Silveira, Rational homotopy theory of fibrations $\ldots \ldots \ldots \ldots 1$

Donald M. Davis, Desuspensions of stunted projective spaces ............ 35

Lou van den Dries, Exponential rings, exponential polynomials and

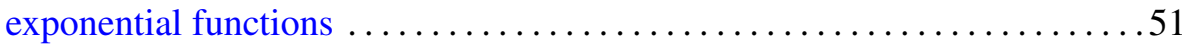

Fred Galvin and Samuel David Shore, Completeness in semimetric

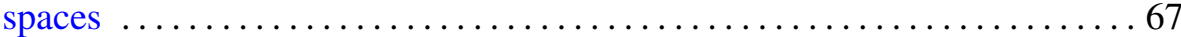

Fereidoun Ghahramani, Compact elements of weighted group algebras . . . 777

Munehiko Itōo, The closed image of a hereditary $M_{1}$-space is $M_{1} \ldots \ldots \ldots 85$

Elvira Laura Livorni, Classification of algebraic surfaces with sectional genus less than or equal to six. I. Rational surfaces .............. 93

H. Alan MacLean, Riesz sets and a theorem of Bochner ............ 115

E. Neher, Jordan triple systems with completely reducible derivation or

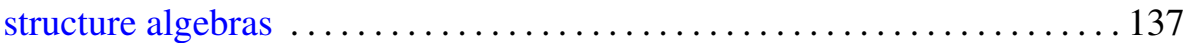

Joe Repka, Shalika's germs for $p$-adic GL( $n)$. I. The leading term $\ldots \ldots \ldots 165$

Joe Repka, Shalika's germs for $p$-adic GL( $n)$. II. The subregular term $\ldots \ldots 173$

Rae Michael Andrew Shortt, Borel density, the marginal problem and isomorphism types of analytic sets $\ldots \ldots \ldots \ldots \ldots \ldots \ldots \ldots \ldots \ldots \ldots \ldots \ldots$

Baruch Solel, The multiplicity functions of invariant subspaces for nonselfadjoint crossed products $\ldots \ldots \ldots \ldots \ldots \ldots \ldots \ldots \ldots \ldots \ldots \ldots . \ldots \ldots$

Su-win Yang, Self-intersection number of immersions and enumeration of nonstable vector bundles

W. M. Zajączkowski, Local solvability of nonstationary leakage problem for ideal incompressible fluid. II 